\title{
On an Iterative Method for Finding a Zero to the Sum of Two Maximal Monotone Operators
}

\author{
Hongwei Jiao ${ }^{1}$ and Fenghui Wang ${ }^{2}$ \\ ${ }^{1}$ School of Mathematical Science, Henan Institute of Science and Technology, Xinxiang 453003, China \\ ${ }^{2}$ Department of Mathematics, Luoyang Normal University, Luoyang 471022, China \\ Correspondence should be addressed to Hongwei Jiao; hongweijiao@126.com and Fenghui Wang; wfenghui@163.com
}

Received 2 March 2014; Revised 18 August 2014; Accepted 19 August 2014; Published 21 August 2014

Academic Editor: Luigi Muglia

Copyright (c) $2014 \mathrm{H}$. Jiao and F. Wang. This is an open access article distributed under the Creative Commons Attribution License, which permits unrestricted use, distribution, and reproduction in any medium, provided the original work is properly cited.

In this paper we consider a problem that consists of finding a zero to the sum of two monotone operators. One method for solving such a problem is the forward-backward splitting method. We present some new conditions that guarantee the weak convergence of the forward-backward method. Applications of these results, including variational inequalities and gradient projection algorithms, are also considered.

\section{Introduction}

It is well known that monotone inclusions problems play an important role in the theory of nonlinear analysis. This problem consists of finding a zero of maximal monotone operators. However, in some examples such as convex programming and variational inequality problems, the operator is needed to be decomposed of the sum of two monotone operators (see, e.g., [1-6]). In this way, one needs to find $x \in \mathscr{H}$ so that

$$
0 \in A x+B x,
$$

where $A$ and $B$ are two monotone operators on a Hilbert space $\mathscr{H}$. To solve such problem, the splitting method, such as Peaceman-Rachford algorithm [7] and Douglas-Rachford algorithm [8], is usually considered. We consider a special case whenever $B: \mathscr{H} \rightarrow 2^{\mathscr{H}}$ is multivalued and $A: \mathscr{H} \rightarrow$ $\mathscr{H}$ is single-valued. A classical way to solve problem (1) under our assumption is the forward-backward splitting (FBS) (see $[2,9])$. Starting with an arbitrary initial $x_{0} \in \mathscr{H}$, the FBS generates a sequence $\left(x_{n}\right)$ satisfying

$$
x_{n+1}=(I+r B)^{-1}(I-r A) x_{n},
$$

where $r$ is some properly chosen real number. Then the FBS converges weakly to a solution of problem (1) whenever such point exists.
On the other hand, we observe that problem (1) is equivalent to the fixed point equation:

$$
(I+r B)^{-1}(I-r A) x=x,
$$

for the single-valued operator $(I+r B)^{-1}(I-r A)$. Moreover, if $r$ is properly chosen, the operator $(I+r B)^{-1}(I-r A)$ should be nonexpansive. Motivated by this assumption, by using the techniques of the fixed point theory for nonexpansive operators, we try to investigate and study various monotone inclusion problems.

The rest of this paper is organized as follows. In Section 2, some useful lemmas are introduced. In Section 3, we consider the modified forward-backward splitting method and prove its weak convergence under some new conditions. In Section 4, some applications of our results in finding a solution of the variational inequality problem are included.

\section{Preliminary and Notation}

Throughout the paper, $I$ denotes the identity operator, $\operatorname{Fix}(S)$ the set of the fixed points of an operator $S$, and $\nabla f$ the gradient of the functional $f: \mathscr{H} \rightarrow \mathbb{R}$. The notation " $\rightarrow$ " denotes strong convergence and “ $\rightarrow$ " weak convergence. Denote by $\omega_{w}\left(x_{n}\right)$ the set of the cluster points of $\left(x_{n}\right)$ in the weak topology (i.e., the set $\left\{x: \exists x_{n_{j}}-x\right\}$, where $\left(x_{n_{j}}\right)$ means a subsequence of $\left.\left(x_{n}\right)\right)$. 
Let $C$ be a nonempty closed convex subset of $\mathscr{H}$. Denote by $P_{C}$ the projection from $\mathscr{H}$ onto $C$; namely, for $x \in \mathscr{H}, P_{C} x$ is the unique point in $C$ with the property

$$
\left\|x-P_{C} x\right\|=\min _{y \in C}\|x-y\| .
$$

It is well-known that $P_{C} x$ is characterized by the inequality

$$
\left\langle x-P_{C} x, z-P_{C} x\right\rangle \leq 0, \quad z \in C .
$$

A single-valued operator $S: \mathscr{H} \rightarrow \mathscr{H}$ is called nonexpansive if

$$
\|S x-S y\| \leq\|x-y\| \quad(\forall x, y \in \mathscr{H})
$$

firmly nonexpansive if

$$
\langle S x-S y, x-y\rangle \geq\|S x-S y\|^{2} \quad(\forall x, y \in \mathscr{H}) ;
$$

and $\kappa$-averaged if there exists a constant $\kappa \in(0,1)$ and a nonexpansive operator $R$ such that $S=(1-\kappa) I+\kappa R$. Firmly nonexpansive operators are $(1 / 2)$-averaged.

Lemma 1 (see [10]). The following assertions hold.

(i) $S$ is $\kappa$-averaged for $\kappa \in(0,1)$ if and only if

$$
\|S x-S y\|^{2} \leq\|x-y\|^{2}-\frac{1-\kappa}{\kappa}\|(I-S) x-(I-S) y\|^{2}
$$

for all $x, y \in \operatorname{dom} S$.

(ii) Assume that $S_{i}$ is $\kappa_{i}$-averaged for $\kappa_{i} \in(0,1), i=1,2$. Then $S_{1} S_{2}$ is $\kappa$-averaged with $\kappa=\kappa_{1}+\kappa_{2}-\kappa_{1} \kappa_{2}$.

The following lemma is known as the demiclosedness principle for nonexpansive mappings.

Lemma 2. Let $C$ be a nonempty closed convex subset of $\mathscr{H}$ and $S$ a nonexpansive operator with $\operatorname{Fix}(S) \neq \emptyset$. If $\left(x_{n}\right)$ is a sequence in $C$ such that $x_{n} \rightarrow x$ and $(I-S) x_{n} \rightarrow y$, then $(I-S) x=y$. In particular, if $y=0$, then $x \in \operatorname{Fix}(S)$. if

A multivalued operator $B: \mathscr{H} \rightarrow 2^{\mathscr{H}}$ is called monotone

$$
\langle u-v, x-y\rangle \geq 0, \quad(\forall u \in B x, v \in B y)
$$

$\kappa$-inverse strongly monotone $(\kappa$-ism), if there exists a constant $\kappa>0$ so that

$$
\langle u-v, x-y\rangle \geq \kappa\|u-v\|^{2}, \quad(\forall u \in B x, v \in B y)
$$

and maximal monotone if it is monotone and its graph $G(B)=\{(x, y): y \in B x\}$ is not properly contained in the graph of any other monotone operator.

In what follows, we shall assume that

(i) $A: \mathscr{H} \rightarrow \mathscr{H}$ is single-valued and $\kappa$-ism;

(ii) $B: \mathscr{H} \rightarrow 2^{\mathscr{H}}$ is multivalued and maximal monotone.
Hereafter, if no confusion occurs, we denote by

$$
J_{r}:=(I+r B)^{-1}
$$

the resolvent of $B$ for any given $r>0$. It is known that $J_{r}$ is single-valued and firmly nonexpansive; moreover $\operatorname{dom}(I+$ $r B)=\mathscr{H}($ see $[11])$.

Lemma 3 (see [12]). For $r>0$, let $T_{r}=J_{r}(I-r A)$. Then

(i) $\operatorname{Fix}\left(T_{r}\right)=(A+B)^{-1}(0)$;

(ii) $T_{r}$ is $(2 \kappa+r) / 4 \kappa$-averaged;

(iii) For $0<r \leq s \leq 2 \kappa, x \in \mathscr{H}$, it follows that

$$
\begin{gathered}
\left\|x-T_{r} x\right\| \leq 2\left\|x-T_{s} x\right\|, \\
\left\|T_{s} x-T_{r} x\right\| \leq\left|1-\frac{r}{s}\right|\left\|x-T_{s} x\right\| .
\end{gathered}
$$

Definition 4. Assume that $\left(x_{n}\right)$ is a sequence in $\mathscr{H}$ and that $\left(\epsilon_{n}\right)$ is a real sequence with $\sum_{n} \epsilon_{n}<\infty$. Then $\left(x_{n}\right)$ is called quasi Fejér monotone w.r.t. $C$, if

$$
\left\|x_{n+1}-z\right\| \leq\left\|x_{n}-z\right\|+\epsilon_{n} \quad(\forall z \in C) .
$$

Lemma 5 (see [13]). Let $C$ be a nonempty closed convex subset of $\mathscr{H}$. If the sequence $\left(x_{n}\right)$ is quasi-Fejér-monotone w.r.t. $C$, then the following hold:

(i) $x_{n} \rightarrow x^{*} \in C$ if and only if $\omega_{w}\left(x_{n}\right) \subseteq C$;

(ii) the sequence $\left(P_{C} x_{n}\right)$ converges strongly;

(iii) if $x_{n} \rightarrow x^{*} \in C$, then $x^{*}=\lim _{n \rightarrow \infty} P_{C} x_{n}$.

\section{Weak Convergence Theorem}

In [10] Combettes considered a modified FBS: for any initial guess $x_{0} \in \mathscr{H}$, set

$$
x_{n+1}=\left(1-\alpha_{n}\right) x_{n}+\alpha_{n} J_{r_{n}}\left(x_{n}-r_{n} A x_{n}\right)+e_{n},
$$

where $\left(\alpha_{n}\right) \subseteq[0,1],\left(r_{n}\right) \subseteq[0,2 \kappa]$ and $\left(e_{n}\right) \subseteq \mathscr{H}$ is computation error. He proved the weak convergence of algorithm (14) provided that

(1) $\underline{\lim }_{n} \alpha_{n}>0$,

(2) $0<\underline{\lim }_{n} r_{n} \leq \overline{\lim }_{n} r_{n}<2 \kappa$,

(3) $\sum_{n}\left\|e_{n}\right\|<\infty$.

We observe that (14) is in fact a Mann-type iteration. In the following we shall prove the convergence of (14) under some sightly weak conditions.

Theorem 6. Suppose the following conditions are satisfied:

(C1) $\varlimsup_{n} \alpha_{n}>0$;

(C2) $0 \leq r_{n} \leq 2 \kappa$;

(C3) $0 \leq \alpha_{n} \leq 4 \kappa /\left(2 \kappa+r_{n}\right)$;

(C4) $\varlimsup_{n}\left(4 \kappa /\left(2 \kappa+r_{n}\right)-\alpha_{n}\right)>0$;

(C5) $\sum_{n}\left\|e_{n}\right\|<\infty$. 
If in addition $\Omega:=(A+B)^{-1}(0) \neq \emptyset$, then the sequence $\left(x_{n}\right)$ generated by (14) converges weakly to $x^{*}:=P_{\Omega} x_{n}$.

Proof. We first show that $\left(x_{n}\right)$ is quasi-Fejér-monotone. Let $T_{n}=J_{r_{n}}\left(I-r_{n} A\right)$. Then It follows from Lemma 3 that $T_{n}$ is $\beta_{n}$-averaged with $\beta_{n}=\left(2 \kappa+r_{n}\right) / 4 \kappa$ and $\Omega=\operatorname{Fix}\left(T_{n}\right)$. Letting $z \in \Omega$, we have that

$$
\begin{aligned}
\left\|x_{n+1}-z\right\| & =\left\|\left(1-\alpha_{n}\right)\left(x_{n}-z\right)+\alpha_{n}\left(T_{n} x_{n}-z\right)+e_{n}\right\| \\
& \leq\left(1-\alpha_{n}\right)\left\|x_{n}-z\right\|+\alpha_{n}\left\|x_{n}-z\right\|+\left\|e_{n}\right\| \\
& =\left\|x_{n}-z\right\|+\left\|e_{n}\right\| .
\end{aligned}
$$

By condition (C5), we conclude that $\left(x_{n}\right)$ is quasi-Fejérmonotone w.r.t $\Omega$.

Next let us show $\omega_{w}\left(x_{n}\right) \subseteq \Omega$. To see this, choose $M>0$ so that

$$
2 \sup _{n \geq 0}\left(\left\|x_{n}-z\right\|\right) \leq M .
$$

Let $R_{n}=\left(1-\alpha_{n}\right) I+\alpha_{n} T_{n}$. Obviously $R_{n}$ is $\alpha_{n} \beta_{n}$-averaged. According to Lemma 1, we deduce that

$$
\begin{aligned}
\left\|x_{n+1}-z\right\|^{2} & =\left\|\left(\left(1-\alpha_{n}\right) I+\alpha_{n} T_{n}\right) x_{n}-z+e_{n}\right\|^{2} \\
\leq & \left\|R_{n} x_{n}-z\right\|^{2}+2\left\langle x_{n+1}-z, e_{n}\right\rangle \\
\leq & \left\|R_{n} x_{n}-z\right\|^{2}+M\left\|e_{n}\right\| \\
\leq & \left\|x_{n}-z\right\|^{2}-\frac{1-\alpha_{n} \beta_{n}}{\alpha_{n} \beta_{n}}\left\|R_{n} x_{n}-x_{n}\right\|^{2}+M\left\|e_{n}\right\| \\
\leq & \left\|x_{n}-z\right\|^{2}-\alpha_{n}\left(\frac{1}{\beta_{n}}-\alpha_{n}\right)\left\|T_{n} x_{n}-x_{n}\right\|^{2} \\
& +M\left\|e_{n}\right\|,
\end{aligned}
$$

which in turn implies that

$$
\sum_{i=0}^{n} \alpha_{i}\left(\frac{1}{\beta_{i}}-\alpha_{i}\right)\left\|T_{i} x_{i}-x_{i}\right\|^{2} \leq\left\|x_{0}-z\right\|^{2}+M \sum_{i=0}^{\infty}\left\|e_{i}\right\|
$$

for all $n \in \mathbb{N}$. Letting $n \rightarrow \infty$ yields

$$
\sum_{n=0}^{\infty} \alpha_{n}\left(\frac{1}{\beta_{n}}-\alpha_{n}\right)\left\|T_{n} x_{n}-x_{n}\right\|^{2}<\infty .
$$

By conditions ( $\mathrm{C} 1)$ and $(\mathrm{C} 4)$, we check that

$$
\liminf _{n \rightarrow \infty} \alpha_{n}\left(\frac{1}{\beta_{n}}-\alpha_{n}\right)>0,
$$

which yields that $\left\|T_{n} x_{n}-x_{n}\right\| \rightarrow 0$. By condition (C2), we find $n_{0} \in \mathbb{N}$ and $r \in(0,2 \kappa]$ so that $r_{n} \geq r$ for all $n \geq n_{0}$. Let $T_{r}=J_{r}(I-r B)$. It then follows from Lemma 3 that

$$
\left\|T_{r} x_{n}-x_{n}\right\| \leq 2\left\|T_{n} x_{n}-x_{n}\right\|
$$

for all $n \geq n_{0}$. Letting $n \rightarrow \infty$, we have $\left\|T_{r} x_{n}-x_{n}\right\| \rightarrow 0$ as $n \rightarrow \infty$. Take $x^{\prime} \in \omega_{w}\left(x_{n}\right)$ and a subsequence $\left(x_{n_{k}}\right)$ of $\left(x_{n}\right)$ such that $x_{n_{k}} \rightarrow x^{\prime}$. Since $T_{r}$ is nonexpansive, applying Lemmas 2 and 3 yields $x^{\prime} \in \operatorname{Fix}\left(T_{r}\right)=\Omega$ and thus $\omega_{w}\left(x_{n}\right) \subseteq$ $\Omega$. By Lemma 5 the proof is complete.

We can also present another condition for the weak convergence of (14).

Theorem 7. Suppose that the following conditions are satisfied:

(C1) $\sum_{n}\left|r_{n+1}-r_{n}\right|<\infty$;

(C2) $0<\lim _{n} r_{n} \leq 2 \kappa$;

(C3) $0 \leq \alpha_{n} \leq 4 \kappa /\left(2 \kappa+r_{n}\right)$;

(C4) $\sum_{n} \alpha_{n}\left(4 \kappa /\left(2 \kappa+r_{n}\right)-\alpha_{n}\right)=\infty$;

(C5) $\sum_{n}\left\|e_{n}\right\|<\infty$.

If in addition $\Omega:=(A+B)^{-1}(0) \neq \emptyset$, then the sequence $\left(x_{n}\right)$ generated by (14) converges weakly to $x^{*}:=P_{\Omega} x_{n}$.

Proof. Compared with the proof of Theorem 6, it suffices to show that $\left\|T_{n} x_{n}-x_{n}\right\| \rightarrow 0$ as $n \rightarrow \infty$. Observe that the estimate

$$
\sum_{n=0}^{\infty} \alpha_{n}\left(\frac{4 \kappa}{2 \kappa+r_{n}}-\alpha_{n}\right)\left\|T_{n} x_{n}-x_{n}\right\|^{2}<\infty
$$

still holds. Let $a_{n}:=\left\|x_{n}-T_{n} x_{n}\right\|$. Then by condition (C4) $\lim _{n} a_{n}=0$. According to Lemma 3 , we have

$$
\begin{aligned}
a_{n+1}= & \left\|\left(1-\alpha_{n}\right)\left(x_{n}-T_{n} x_{n}\right)+\left(T_{n} x_{n}-T_{n+1} x_{n+1}\right)+e_{n}\right\| \\
\leq & \left(1-\alpha_{n}\right)\left\|x_{n}-T_{n} x_{n}\right\|+\left\|T_{n} x_{n}-T_{n+1} x_{n+1}\right\| \\
& +\left\|e_{n}\right\| \\
\leq & \left(1-\alpha_{n}\right)\left\|x_{n}-T_{n} x_{n}\right\|+\left\|T_{n+1} x_{n}-T_{n+1} x_{n+1}\right\| \\
& +\left\|T_{n} x_{n}-T_{n+1} x_{n}\right\|+\left\|e_{n}\right\| \\
\leq & \left(1-\alpha_{n}\right)\left\|x_{n}-T_{n} x_{n}\right\|+\left\|x_{n}-x_{n+1}\right\| \\
& +\left\|T_{n} x_{n}-T_{n+1} x_{n}\right\|+\left\|e_{n}\right\| \\
= & \left(1-\alpha_{n}\right)\left\|x_{n}-T_{n} x_{n}\right\|+\left\|\alpha_{n}\left(x_{n}-T_{n} x_{n}\right)-e_{n}\right\| \\
& +\left|1-\frac{r_{n+1}}{r_{n}}\right|\left\|x_{n}-T_{n} x_{n}\right\|+\left\|e_{n}\right\| \\
\leq & \left(1-\alpha_{n}\right)\left\|x_{n}-T_{n} x_{n}\right\|+\alpha_{n}\left\|x_{n}-T_{n} x_{n}\right\| \\
& +\left|1-\frac{r_{n+1}}{r_{n}}\right|\left\|x_{n}-T_{n} x_{n}\right\|+2\left\|e_{n}\right\| \\
\leq & a_{n}+M\left(\left|r_{n}-r_{n+1}\right|+\left\|e_{n}\right\|\right),
\end{aligned}
$$

where $M>0$ is properly chosen real number. Then, for any given $p \in \mathbb{N}$, we arrive at

$$
a_{n+p} \leq a_{n}+M \sum_{i=n}^{\infty}\left(\left|r_{i}-r_{i+1}\right|+\left\|e_{i}\right\|\right) .
$$


Conditions (C1) and (C5) imply that $\lim _{n \rightarrow \infty} a_{n}$ exists and therefore $\left\|x_{n}-T_{n} x_{n}\right\| \rightarrow 0$ as $n \rightarrow \infty$. Hence the proof is complete.

Applying Theorem 7, one can easily get the following.

Corollary 8. Suppose that the following conditions are satisfied:

(1) $0<r<2 \kappa$,

(2) $0 \leq \alpha_{n} \leq 4 \kappa /(2 \kappa+r)$;

(3) $\sum_{n} \alpha_{n}\left(4 \kappa /(2 \kappa+r)-\alpha_{n}\right)=\infty$,

(4) $\Omega:=(A+B)^{-1}(0) \neq \emptyset$.

Then the sequence $\left(x_{n}\right)$ generated by

$$
x_{n+1}=\left(1-\alpha_{n}\right) x_{n}+\alpha_{n} J_{r}\left(x_{n}-r A x_{n}\right)
$$

converges weakly to some point in $\Omega$.

Remark 9. Corollary 8 implies that our condition is slightly weaker than that of Combettes' whenever the sequence $\left(r_{n}\right)$ approaches to some constant.

\section{Application}

Let $C$ be a nonempty closed convex subset of $\mathscr{H}$. A variational inequality problem (VIP) is formulated as a problem of finding a point $x^{*} \in C$ with the property

$$
\left\langle A x^{*}, z-x^{*}\right\rangle \geq 0, \quad \forall z \in C,
$$

where $A: \mathscr{H} \rightarrow \mathscr{H}$ is a nonlinear operator. We shall denote by $\Omega$ the solution set of VIP (26). One method for solving VIP is the projection algorithm which generates, starting with an arbitrary initial $x_{0} \in \mathscr{H}$, a sequence $\left(x_{n}\right)$ satisfying

$$
x_{n+1}=P_{C}\left(x_{n}-r A x_{n}\right) \text {, }
$$

where $r$ is properly chosen real number. If, in addition, $A$ is $\kappa$ ism, then the iteration (27) with $0<r<2 \kappa$ converges weakly to a point in $\Omega$, whenever such point exists.

Let $B$ be the normal cone for $C$, that is, $B:=\{w \in \mathscr{H}:\langle x-$ $z, w\rangle \geq 0, \forall z \in C\}$. By [14, Theorem 3], VIP (26) is equivalent to finding a zero of the maximal monotone operator $A+B$. Recalling $P_{C}=J_{r}$ for any $r>0$, we thus can apply the previous results to get the following.

Corollary 10. Suppose the following conditions are satisfied:

$$
\begin{aligned}
& \text { (C1) } \varlimsup_{n} \alpha_{n}>0 \\
& \text { (C2) } 0 \leq r_{n} \leq 2 \kappa \\
& \text { (C3) } 0 \leq \alpha_{n} \leq 4 \kappa /\left(2 \kappa+r_{n}\right) \\
& \text { (C4) } \varlimsup_{n}\left(4 \kappa /\left(2 \kappa+r_{n}\right)-\alpha_{n}\right)>0 .
\end{aligned}
$$

Then the sequence $\left(x_{n}\right)$ generated by

$$
x_{n+1}=\left(1-\alpha_{n}\right) x_{n}+\alpha_{n} P_{C}\left(x_{n}-r_{n} A x_{n}\right)
$$

converges weakly to $x^{*}:=P_{\Omega} x_{n}$, whenever such point exists.
Corollary 11. Suppose that the following conditions are satisfied:

(C1) $\sum_{n}\left|r_{n+1}-r_{n}\right|<\infty$;

(C2) $0<\lim _{n} r_{n} \leq 2 \kappa$;

(C3) $0 \leq \alpha_{n} \leq 4 \kappa /\left(2 \kappa+r_{n}\right)$;

(C4) $\sum_{n} \alpha_{n}\left(4 \kappa /\left(2 \kappa+r_{n}\right)-\alpha_{n}\right)=\infty$.

Then the sequence $\left(x_{n}\right)$ generated by

$$
x_{n+1}=\left(1-\alpha_{n}\right) x_{n}+\alpha_{n} P_{C}\left(x_{n}-r_{n} A x_{n}\right)
$$

converges weakly to $x^{*}:=P_{\Omega} x_{n}$, whenever such point exists.

Consider the optimization problem of finding a point $x^{*}$ with the property

$$
x^{*} \in \arg \min _{x \in C} f(x),
$$

where $f: \mathscr{H} \rightarrow \mathbb{R}$ is a convex and differentiable function. The gradient projection algorithm (GPA) generates a sequence $\left(x_{n}\right)$ by the iterative procedure

$$
x_{n+1}=P_{C}\left(x_{n}-r \nabla f\left(x_{n}\right)\right),
$$

where $x_{0} \in \mathscr{H}$ and $r$ is a positive parameter. If, in addition, $\nabla f$ is $(1 / \kappa)$-Lipschitz continuous, that is, for any $x, y \in \mathscr{H}$,

$$
\|\nabla f(x)-\nabla f(y)\| \leq \frac{1}{\kappa}\|x-y\|,
$$

then the GPA with $0<r<2 \kappa$ converges weakly to a minimizer of $f$ onto $C$, if such minimizers exist (see, e.g., [15, Corollary 4.1]). Denote by $\Omega$ the solution set of the variational inequality

$$
\langle\nabla f(x), z-x\rangle \geq 0, \quad z \in C .
$$

According to [16, Lemma 5.13], we have $\Omega=\arg \min _{x \in C} f(x)$. Further, if $\nabla f$ is $(1 / \kappa)$-Lipschitz continuous, then it is also $\kappa$ ism (see [17, Corollary 10]). Thus, we can apply the previous results by letting $A=\nabla f$.

Corollary 12. Assume that $f: \mathscr{H} \rightarrow \mathbb{R}$ is convex and differentiable with $(1 / \kappa)$-Lipschitz-continuous gradient $\nabla f$ and that
(C1) $\varlimsup_{n} \alpha_{n}>0$;
(C2) $0 \leq r_{n} \leq 2 \kappa$;
(C3) $0 \leq \alpha_{n} \leq 4 \kappa /\left(2 \kappa+r_{n}\right)$;
(C4) $\overline{\lim }_{n}\left(4 \kappa /\left(2 \kappa+r_{n}\right)-\alpha_{n}\right)>0$.

Then the sequence $\left(x_{n}\right)$ generated by

$$
x_{n+1}=\left(1-\alpha_{n}\right) x_{n}+\alpha_{n} P_{C}\left(x_{n}-r_{n} \nabla f\left(x_{n}\right)\right)
$$

converges weakly to $x^{*}:=P_{\Omega} x_{n}$, whenever such point exists.

Corollary 13. Assume that $f: \mathscr{H} \rightarrow \mathbb{R}$ is convex and differentiable with $(1 / \kappa)$-Lipschitz-continuous gradient $\nabla f$ and that 


$$
\begin{aligned}
& \text { (C1) } \sum_{n}\left|r_{n+1}-r_{n}\right|<\infty \\
& \text { (C2) } 0<\lim _{n} r_{n} \leq 2 \kappa \\
& \text { (C3) } 0 \leq \alpha_{n} \leq 4 \kappa /\left(2 \kappa+r_{n}\right) \\
& \text { (C4) } \sum_{n} \alpha_{n}\left(4 \kappa /\left(2 \kappa+r_{n}\right)-\alpha_{n}\right)=\infty
\end{aligned}
$$

Then the sequence $\left(x_{n}\right)$ generated by

$$
x_{n+1}=\left(1-\alpha_{n}\right) x_{n}+\alpha_{n} P_{C}\left(x_{n}-r_{n} \nabla f\left(x_{n}\right)\right)
$$

converges weakly to $x^{*}:=P_{\Omega} x_{n}$, whenever such point exists.

\section{Conflict of Interests}

The authors declare that there is no conflict of interests regarding the publication of this paper.

\section{Acknowledgments}

The authors would like to express their sincere thanks to the anonymous referees and editors for their careful review of the paper and the valuable comments, which have greatly improved the earlier version of this paper. This work is supported by the National Natural Science Foundation of China (Grant nos. 11301253 and 11271112), the Basic and Frontier Project of Henan (no. 122300410268), and the Science and Technology Key Project of Education Department of Henan Province (14A110024).

\section{References}

[1] J. Eckstein and D. P. Bertsekas, "On the Douglas-Rachford splitting method and the proximal point algorithm for maximal monotone operators," Mathematical Programming, vol. 55, no. 3, pp. 293-318, 1992.

[2] P. L. Lions and B. Mercier, "Splitting algorithms for the sum of two nonlinear operators," SIAM Journal on Numerical Analysis, vol. 16, no. 6, pp. 964-979, 1979.

[3] T. Pennanen, "A splitting method for composite mappings," Numerical Functional Analysis and Optimization, vol. 23, no. 78, pp. 875-890, 2002.

[4] J. E. Spingarn, "Applications of the method of partial inverses to convex programming: decomposition," Mathematical Programming, vol. 32, no. 2, pp. 199-223, 1985.

[5] P. Tseng, "Further applications of a splitting algorithm to decomposition in variational inequalities and convex programming," Mathematical Programming, vol. 48, pp. 249-263, 1990.

[6] P. Tseng, "Applications of a splitting algorithm to decomposition in convex programming and variational inequalities," SIAM Journal on Control and Optimization, vol. 29, no. 1, pp. 119-138, 1991.

[7] D. W. Peaceman and J. Rachford, "The numerical solution of parabolic and elliptic differential equations," Society for Industrial and Applied Mathematics, vol. 3, no. 1, pp. 28-41, 1955.

[8] J. Douglas and H. H. Rachford, "On the numerical solution of heat conduction problems in two or three space variables," Transactions of the American Mathematical Society, vol. 82, pp. 421-439, 1956.

[9] G. B. Passty, "Ergodic convergence to a zero of the sum of monotone operators," Journal of Mathematical Analysis and Applications, vol. 72, no. 2, pp. 383-390, 1979.
[10] P. L. Combettes, "Solving monotone inclusions via compositions of nonexpansive averaged operators," Optimization, vol. 53, no. 5-6, pp. 475-504, 2004.

[11] R. T. Rockafellar, "Monotone operators and the proximal point algorithm," SIAM Journal on Control and Optimization, vol. 14, no. 5, pp. 877-898, 1976.

[12] G. López, V. Martín-Márquez, F. Wang, and H.-K. Xu, "Forward-backward splitting methods for accretive operators in Banach spaces," Abstract and Applied Analysis, vol. 2012, Article ID 109236, 25 pages, 2012.

[13] D. Butnariu, Y. Censor, and S. Reich, Quasi-Fejérian Analysis of Some Optimization Algorithms, Inherently Parallel Algorithms in Feasibility and Optimization and Their Applications, Elsevier Science Publishers, Amsterdam, The Netherlands, 2001.

[14] R. T. Rockafellar, "On the maximality of sums of nonlinear monotone operators," Transactions of the American Mathematical Society, vol. 149, pp. 75-88, 1970.

[15] C. Byrne, "A unified treatment of some iterative algorithms in signal processing and image reconstruction," Inverse Problems, vol. 20, no. 1, pp. 103-120, 2004.

[16] H. W. Engl, M. Hanke, and A. Neubauer, Regularization of Inverse Problems, Kluwer Academic Publishers Group, Dordrecht, The Netherlands, 1996.

[17] J. B. Baillon and G. Haddad, "Quelques propriétés des opérateurs angle-bornés et n-cycliquement monotones," Israel Journal of Mathematics, vol. 26, no. 2, pp. 137-150, 1977. 


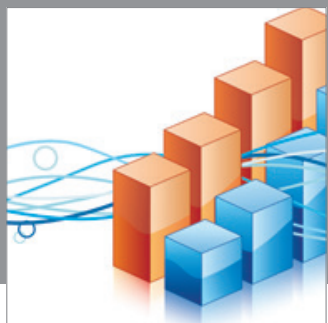

Advances in

Operations Research

mansans

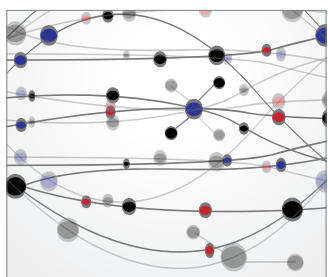

The Scientific World Journal
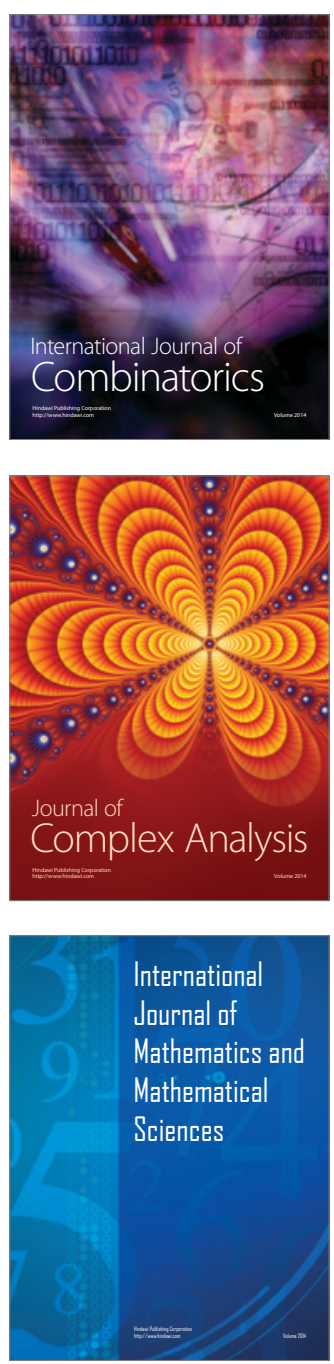
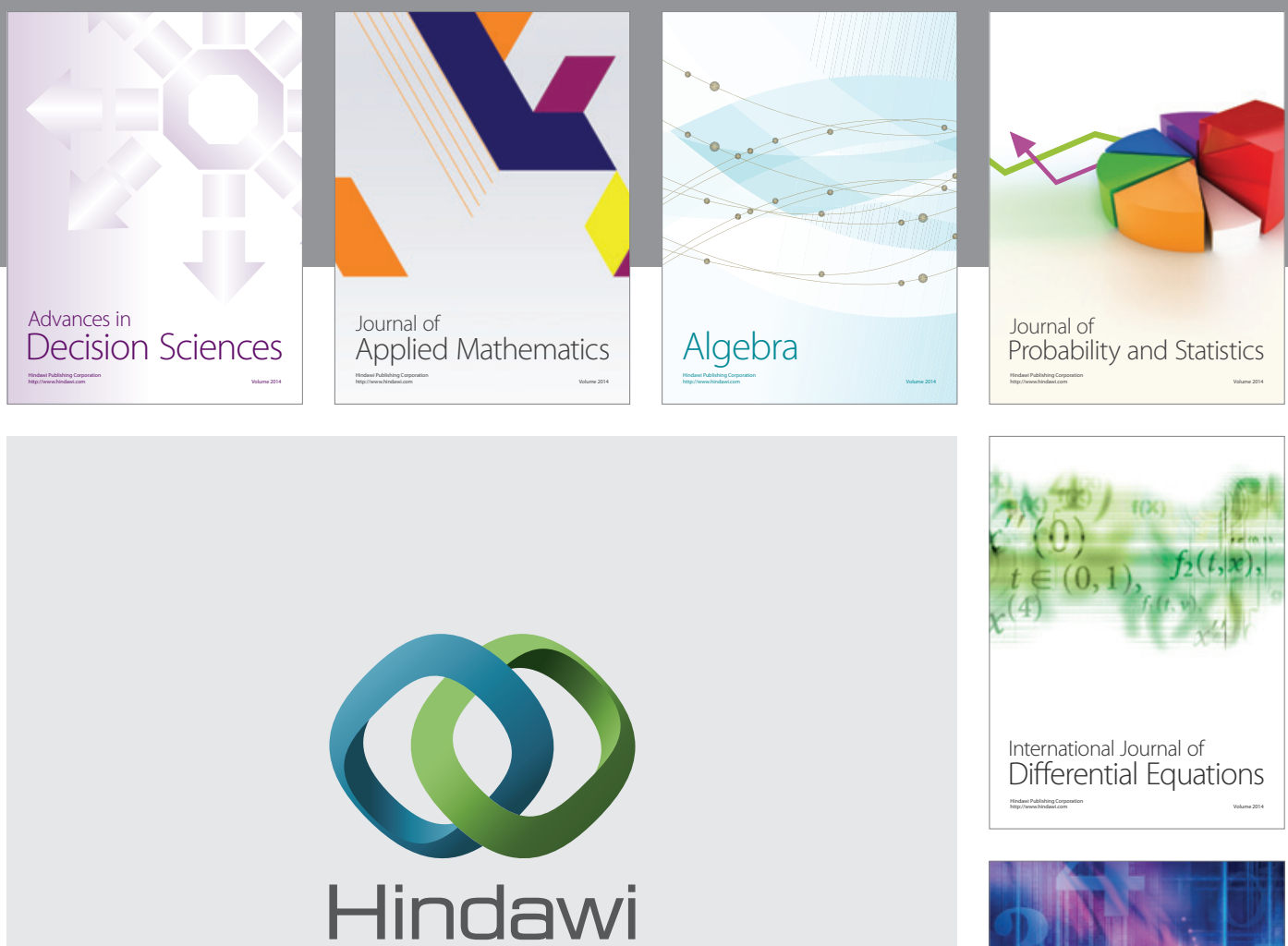

Submit your manuscripts at http://www.hindawi.com
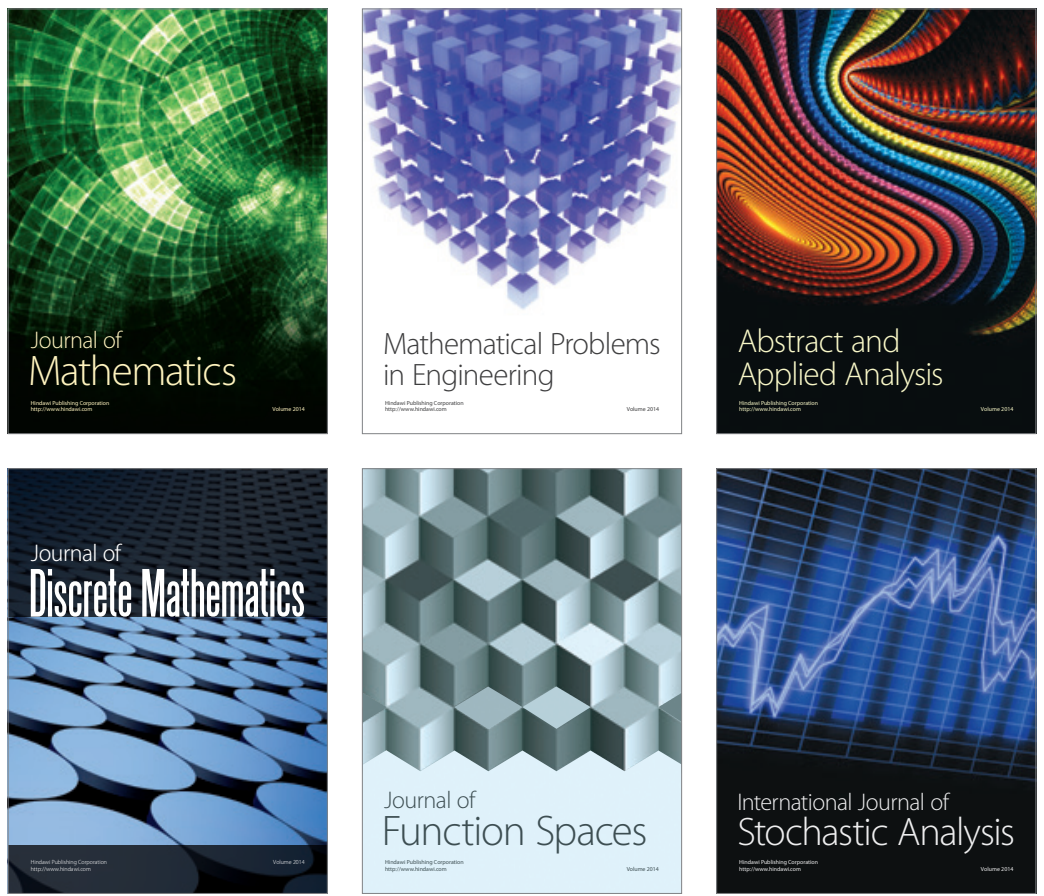

Journal of

Function Spaces

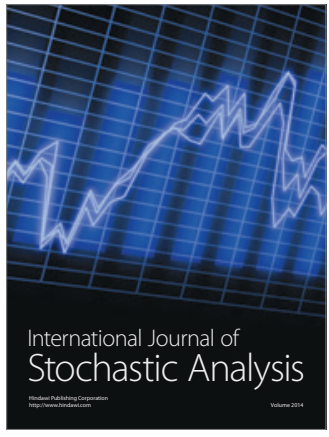

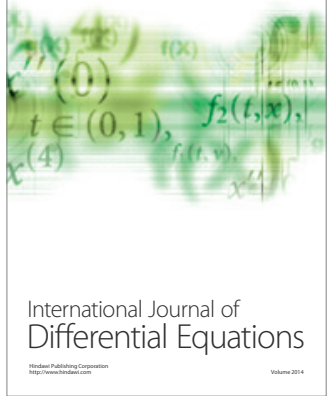
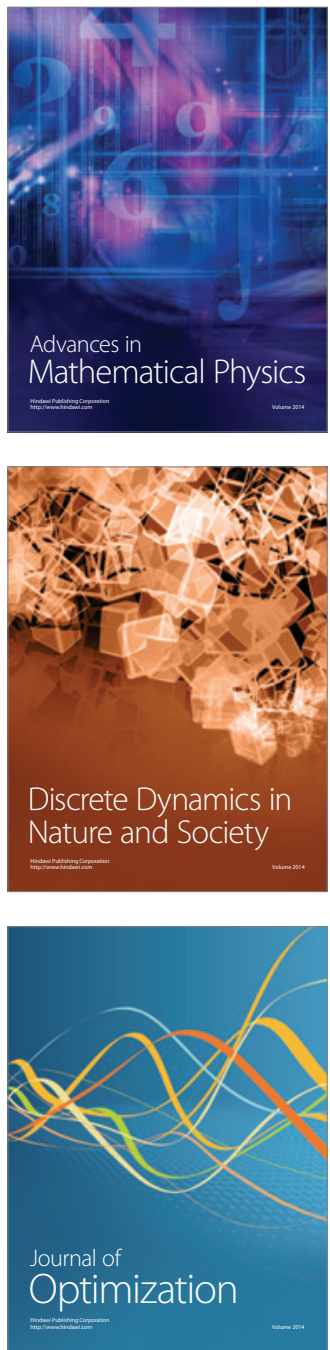